\title{
Characterization of polypropylene pulsating heat stripes: effects of orientation, working fluid, and loop geometry
}

\author{
Oguzhan Der, Ali Ahmed Alqahtani ${ }^{1}$ \\ Laboratory of Technical Physics, University of Liverpool, Brownlow Hill, Liverpool L69 \\ 3GH, United Kingdom \\ Marco Marengo \\ School of Computing, Engineering and Mathematics, University of Brighton, Lewes Road, \\ Brighton BN2 4GJ, United Kingdom \\ Volfango Bertola* \\ Laboratory of Technical Physics, University of Liverpool, Brownlow Hill, Liverpool L69 \\ 3GH, United Kingdom
}

\begin{abstract}
A parametric analysis of the thermal performance of flat polyprpylene pulsating heat pipes (PHPs) is presented. In particular, the thermal performance was charachterized for pulsating heat pipes with different number of turns of the serpentine channel, different orientations with respect to gravity, and containing different heat transfer fluids. The dependence of polymeric PHPs performance on design parameters is poorly understood to date. The development of polymeric PHPs charachterized by high mechanical flexibility will have significant impact on thermal management of smartphones, portable electronics, and deployable systems such as cube satellites. Several prototype PHPs with different number of turns of the serpentine channel were fabricated bonding together three polypropylene sheets by selective transmission laser welding, after cutting out a serpentine channel in the central sheet. The thermal performance of the devices was charachterized by supplying an ascending/descending stepped thermal power ramp to the evaporator, and measuring the corresponding equivalent thermal resistance between the evaporator and the condenser.
\end{abstract}

Keywords: Pulsating heat pipes, Plastic heat pipe, Selective transmission laser welding

\footnotetext{
${ }^{*}$ Corresponding author

Email address: Volfango.Bertola@liverpool.ac.uk (Volfango Bertola)

${ }^{1}$ Permanent address: Mechanical Engineering Department, College of Engineering, King Khalid University, Abha 61413 Asir, Saudi Arabia.
} 


\section{Introduction}

The increasing technology challenges posed by volumetric density scaling in smart systems integration and functional electronic packaging [1] drive the interest in high-performace, compact heat transfer devices for thermal man-

5 agement of computers, data centres, smartphones, high power electronics, and cooling boards. In this context, passive two-phase capillary driven loops, such as pulsating heat pipes (PHPs) are a very promising, simple and cost effective technology compared to other heat transport devices [2, 3].

A pulsating heat pipe consists of a small diameter tube arranged in a ser10 pentine or multi-turn structure and evacuated to a high-vacuum level $\left(10^{-3}\right.$ mBar). A working fluid free from non-condensable gases and with thermophysical properties suitable to maximize the heat transfer is then fed to the system, only partially filling the device. Once the PHP is sealed, the working fluid circulates alternating liquid slugs and vapour plugs due to the dominant

15 effect of capillary forces with respect to the buoyancy forces. The fluid motion as well as the flow pattern transitions are rather chaotic, however the system is able to reach a pseudo-steady state over a wide range of operating conditions [4. The pressure fluctuations inside the PHP drive a self-excited oscillating motion of liquid plugs and vapour bubbles [5], significantly enhancing convec20 tion and consequently promoting the heat transfer of both sensible and latent heat between a thermal source (evaporator) and a thermal sink (condenser) [6. The advantages of PHPs with respect to standard heat transfer technologies, such as purely conductive systems or single phase forced loops, are mainly related to the exploitation of the high heat transfer rates associated with the

25 phase change phenomenon, the compactness and the possibility to be operated passively, avoiding the use of pumps and gravity.

To address the demand of heat transfer devices characterized by low weight, small unit thickness, low cost, and high mechanical flexibility, it was recently proposed to embed the PHP serpentine channel into a composite polypropy-

30 lene sheet [7, 8. The concept is to cut out the serpentine channel in a black polypropylene sheet, sandwiched between two transparent sheets of the same material and bonded together by selective transmission laser welding [9, 10]. Due to the small thickness of the polymer sheet, the PHP channel has a large aspect ratio, resulting into so-called "pulsating heat stripes" (PHS), which can

35 be seen in practice as an engineered composite polymer sheet with enhanced thermal conductivity [11, 12]. The development of polymeric PHPs charachterized by high mechanical flexibility will have significant impact on thermal management of smartphones, portable electronics, and deployable systems such as cube satellites.

40 The liquid and gas diffusion through the polymer and the billowing of the flexible material due to pressure differentials are two important technical issues affecting pulsating heat pipes built with polymeric materials. Very recently, a 
flexible pulsating heat pipe was fabricated by thermally sintering a multilayer polymer film including an aluminum layer acting as gas barrier on the two sides 45 of a low-density polyethylene sheet cut into a closed-loop PHP shape [13. To minimize diffusion of noncondensable gases through the seal, an indium coating was deposited on the heat pipe perimeter.

The list of parameters influencing the PHP effectiveness is quite long, the most important ones being the working fluid, the applied heat flux, the filling ratio, the two-phase flow distribution, the liquid film thickness, the characteristics of the fluid oscillations, the gravity level, and the geometrical features, just to mention the most relevant ones [14, 15. Whilst there seems to be a wide consensus on the optimal value of the filling ratio (50-60\%), the effect of many other parameters is poorly understood or strongly case-dependent. The PHP

55 geometrical parameters impact in complex way on the PHP behaviour. The overall length of the channel, the number of turns, the evaporation, adiabatic and condenser section lengths, the radius of the channel turns, have rather intricate and not well understood effects on the PHP behaviour. Furthermore, increasing the number of turns improves the operation stability, and allows to 60 dissipate higher power inputs with almost comparable evaporator temperatures, resulting into a decrease of the thermal resistance.

Because the development of polymeric pulsating heat pipes is still in its infancy, to date there is no systematic assessment of the effect of design parameters on their performance. To partially address this gap in the current understanding of polymeric pulsating heat pipes, the present work reports the results of an experimental investigation of the effects of orientation, working fluid and loop geometry on the thermal performance of a flat polypropylene pulsating heat pipe.

\section{PHS design}

The pulsating heat pipes (or PHS) used in the present study were fabricated using rectangular polypropylene sheets having a length of $250 \mathrm{~mm}$ and a width of $100 \mathrm{~mm}$. First, the serpentine channel was cut-out in a black sheet $(0.7 \mathrm{~mm}$ thickness) using a commercial laser cutter (HPC Laser LS1290 Pro). Then, the channel was sandwiched between two transparent sheets $(0.4 \mathrm{~mm}$ thickness laser welding using a nanosecond pulsed fibre laser (SPI Lasers G4 HS-L 20W) 7, [8, 11, 12]. The resulting composite polypropylene sheet had an overall thickness of $1.5 \mathrm{~mm}$.

The most important design parameters of a pulsating heat pipe are the so hydraulic diameter, $D_{H}=2 W t /(W+t)$, where $W$ is the channel width and $t$ its height, and the number of turns of the serpentine channel. To ensure the fluid circulation is driven by capillary forces instead of buoyancy, one must set an upper limit to the Bond number $B o=g\left(\rho_{l}-\rho_{v}\right) D_{H}^{2} / \sigma$, where $g$ is gravity, $\rho_{l}$ and $\rho_{v}$ are, respectively, the liquid and the vapour densities, and $\sigma$ is the surface

85 tension. Studies of capillary flow [16] and flooding in vertical up-flow [17] and of heat transfer in confined spaces under a variety of conditions [18, 19, 20] suggest 
Table 1: Fluid properties at ambient temperature and at saturation temperature.

\begin{tabular}{llllllll}
\hline & $\begin{array}{l}\mathrm{T} \\
{\left[{ }^{\circ} \mathrm{C}\right]}\end{array}$ & $\begin{array}{l}\rho_{l} \\
{\left[\mathrm{~kg} / \mathrm{m}^{3}\right]}\end{array}$ & $\rho_{v}$ & $H_{l v}$ & $\sigma$ & $\theta$ & $P_{v}$ \\
& 20 & 998 & 0.008 & 2453 & 72 & 85 & 2.34 \\
& {$[\mathrm{~kJ} / \mathrm{kgK}]$} & {$[\mathrm{mN} / \mathrm{m}]$} & {$\left[{ }^{\circ}\right]$} & {$[\mathrm{kPa}]$} \\
\hline water & 20 & 959 & 0.6 & 2256 & 59 & 85 & 101 \\
water & 99 & 796 & 0.145 & 919 & 24 & 0 & 5.83 \\
ethanol & 20 & 789 & 1.87 & 846 & 17 & 0 & 100 \\
ethanol & 78 & 737 & 3.46 & 95 & 12.7 & 75 & 27 \\
FC-72 & 20 & 1688 & 3.7 & 7.8 & 75 & 100 \\
FC-72 & 56 & 1594 & 13.1 & 88 & 7.8 & &
\end{tabular}

that capillary effects are significant for channels having hydraulic diameters such that the $B o<4$ [21, 22]. There is also experimental evidence of a lower limit for PHP operation, $B o>0.4$ [23. This results into the following lower and upper limits for the hydraulic diameter:

$$
0.63 \sqrt{\frac{\sigma}{g\left(\rho_{l}-\rho_{v}\right)}} \leq D_{H} \leq 2 \sqrt{\frac{\sigma}{g\left(\rho_{l}-\rho_{v}\right)}}
$$

According to Eq. (1), the range of acceptable hydraulic diameters depends only of the fluid properties, which in turn depend on temperature. In the present work, three commonly used heat transfer fluids (water, ethanol, and perfluorohexane FC-72) were considered, and their properties are listed in Table

95 1. All properties were taken from the reference literature, except the contact angle, which was measured from side view images of liquid drops deposited on the same polyropylene sheets used to fabricate the PHP using a specific digital image processing method [24, 25].

Because one dimension of the channel cross-section is fixed $(t=0.7 \mathrm{~mm})$, the hydraulic diameter and the Bond number for a given fluid depend only on the channel width. Figure 1 1 displays the Bond number as a function of the channel width, for the three fluids considered, with thermophysical properties evaluated at ambient temperature $\left(20^{\circ} \mathrm{C}\right)$ and at the boiling point, i.e. the limits of the temperature range where the fluid is in the liquid state. This figure 105 shows that in the case of FC-72 and ethanol, the Bond number falls within the range suitable to PHP operation for channel widths between 2.5 and $10 \mathrm{~mm}$, while the Bond numbers relative to water are too low for PHP operation, for any channel width.

The upper and lower limits on the Bond number, and consequently those on ${ }_{110}$ the hydraulic diameter (Eq. 1), however, were determined based on experiments in metallic or glass capillary tubes, where the liquid is in contact with materials characterised by high surface energy hence high wettability [26]. In the case of polypropylene, the surface energy of the channel walls is significantly lower, therefore the capillary force at thermodynamic equilibrium reduces according

115 to the Young-Laplace equation, and the Bond number should be calculated as $B o=g\left(\rho_{l}-\rho_{v}\right) D_{H}^{2} / \sigma \cos \theta$, where $\theta$ is the equilibrium contact angle of the fluid 

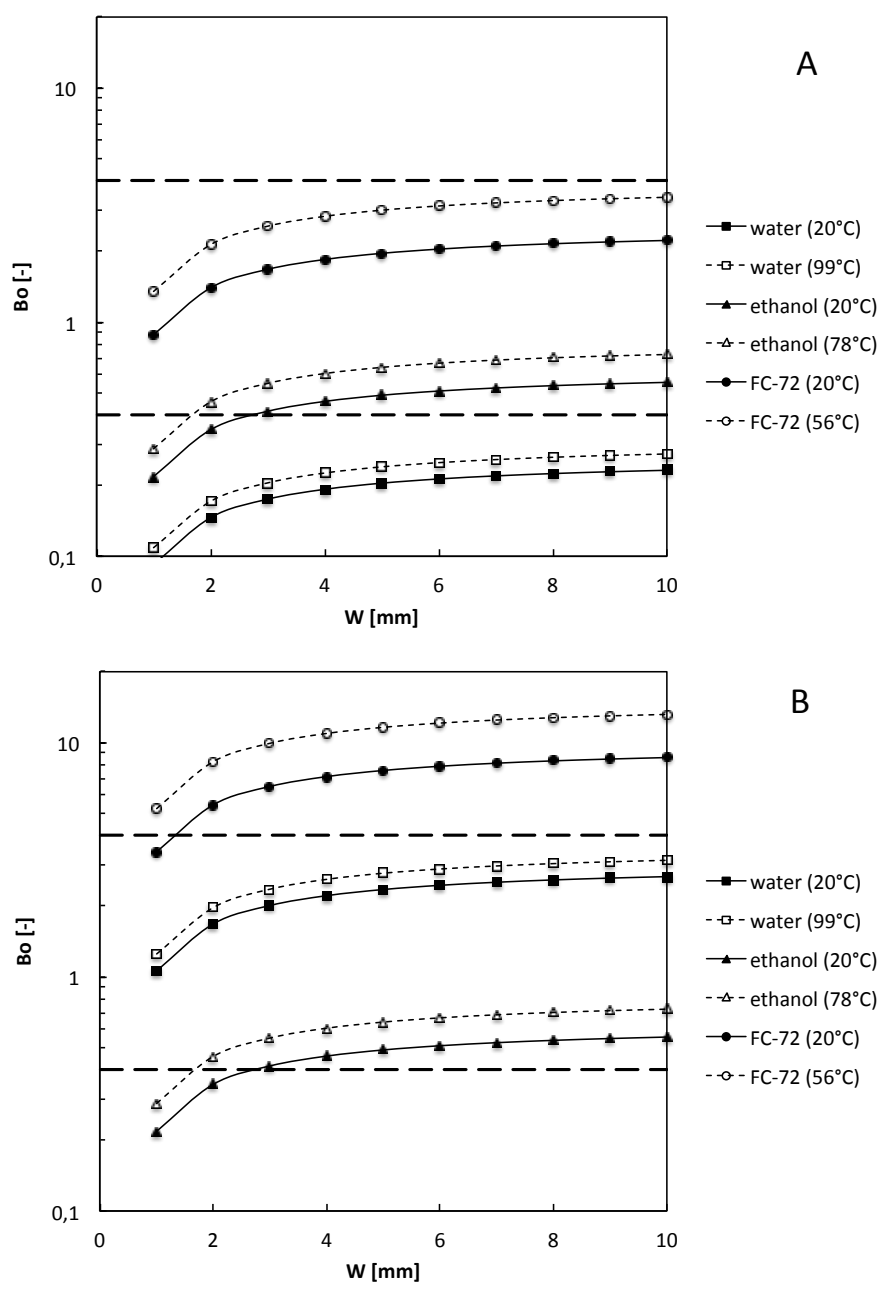

Figure 1: Bond number for water, ethanol and FC-72 at ambient temperature and at the boiling point, plotted as a function of the channel width without considering wettability (a) and including the effect of wettability (b). The long-dashed horizontal lines indicate the lower and upper limits on the Bond number.

on polypropylene. Accordingly, the range of acceptable hydraulic diameters for PHP operation becomes:

$$
0.63 \sqrt{\frac{\sigma \cos \theta}{g\left(\rho_{l}-\rho_{v}\right)}} \leq D_{H} \leq 2 \sqrt{\frac{\sigma \cos \theta}{g\left(\rho_{l}-\rho_{v}\right)}}
$$

Taking into account the fluid wettability, the Bond numbers corresponding 120 to the three fluids considered change as shown in Figure $1 \mathrm{p}$, which suggests in the case of water and ethanol the Bond number falls within the range suitable 
to PHP operation for channel widths between 2.5 and $10 \mathrm{~mm}$, while the Bond numbers calculated for FC-72 are higher than the upper limit $(B o=4)$. This implies that in the case of FC-72 buoyancy exceeds significantly the capillary driving force, therefore the PHP should operate as a thermosyphon, i.e. driven by natural convection.

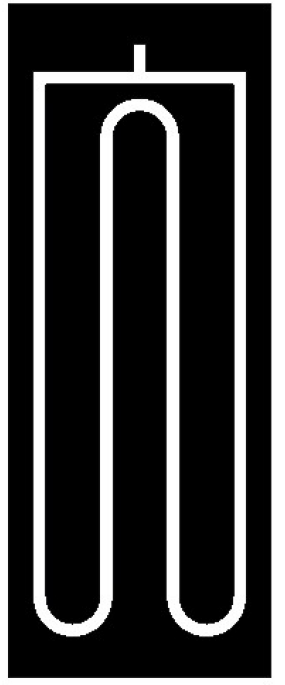

(a)

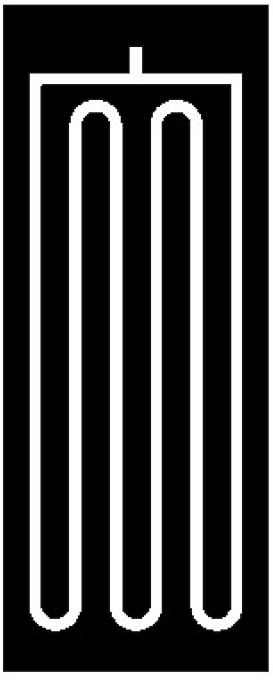

(b)

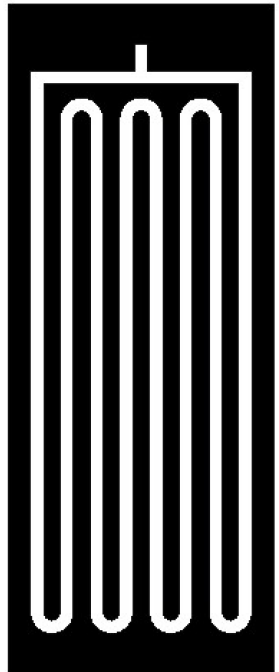

(c)

Figure 2: Prototype pulsating heat stripes with 3-turns (a), 5-turns (b), and 7-turns (c) serpentine channel.

Another constraint on the channel width is due by the relatively low elastic modulus of polypropylene. If the channel width is large, the thin $(0.4 \mathrm{~mm})$ polypropylene wall changes its shape according to the pressure difference between the heat transfer fluid and the atmosphere. This affects both the pressure level during operation and the hydraulic diameter, as shown by preliminary experiments conducted on a polypropylene PHP/PHS having a channel width of $9 \mathrm{~mm}[8$.

In conclusion, a channel width $W=5 \mathrm{~mm}$ was selected to meet all of the above constraints, corresponding to a hydraulic diameter $D_{H}=1.23 \mathrm{~mm}$. As for the number of turns, three pulsating heat pipe designs were produced with three, five, and seven turns, respectively, as illustrated in Figure 2. On the condenser side, the main loop is connected to a short auxiliary channel where a small hole in one of the channel walls enables fitting a valve and a pressure 


\section{Experimental setup and procedure}

\subsection{Experimental setup}

Figure 3 shows the schematic layout of the experimental setup. The PHS units were mounted on a support frame, clamped in correspondence of the evaporator zone and of the condenser zone. Each side of the evaporator clamp consisted of a rectangular plywood plate $(15 \mathrm{~cm}$ length, $5 \mathrm{~cm}$ width, $1.5 \mathrm{~cm}$ thickness) insulated on one side by a $1 \mathrm{~cm}$ layer of Superwool 607HT (Thermal Ceramics Ltd.), a material with thermal conductivity $\leq 0.05 \mathrm{~W} / \mathrm{mK}$. The insulating layer was shaped to host a $100 \mathrm{~W}$ ceramic heater and a flush-mounted copper plate $(10 \mathrm{~cm}$ length, $4 \mathrm{~cm}$ width), to distribute the heat supply uniformly on the PHP/PHS evaporator surface. Heat sink paste was used to minimise the contact resistance between the ceramic heater and the copper plate. The heaters were connected to a regulated DC power supply (Circuit Specialists CSI 12001X) to enable a fine control of the power input.

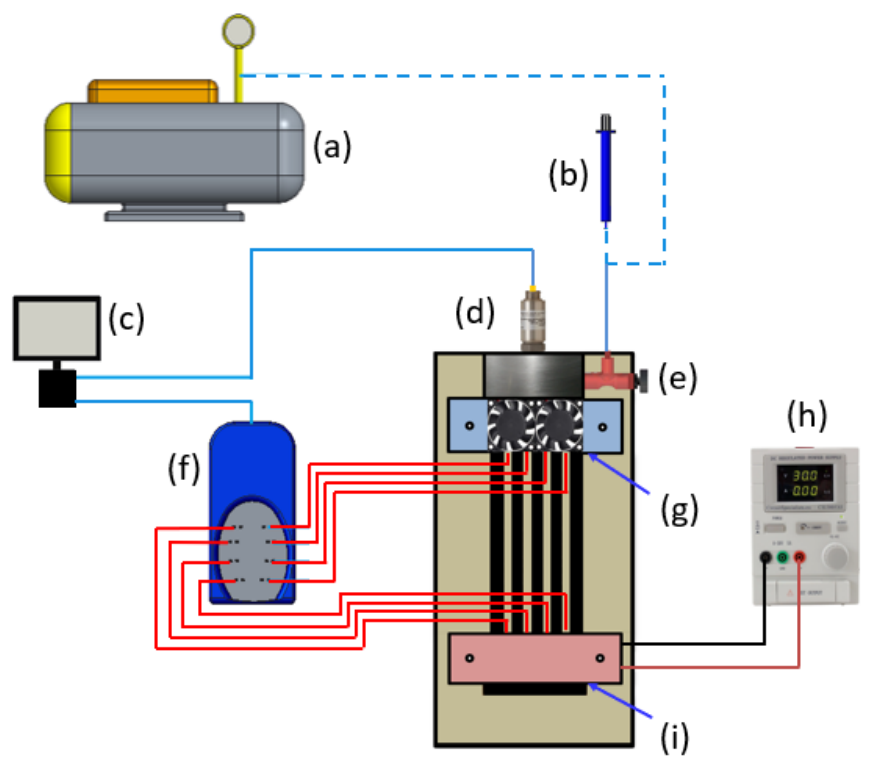

Figure 3: Schematic layout of the experimental setup: (a) vacuum pump, (b) syringe, (c) data logger and PC, (d) pressure transducer, (e) micro-metering valve, (f) thermocouples data logger, (g) condenser fans, (h) power supply, and (i) electric heaters.

In the condenser section, the PHS were clamped between two aluminium plates coated with a thin layer of heat sink paste; two fan-assisted heat sinks (Malico) were mounted on the external plate. The evaporator and the condenser sections had an identical length of $4 \mathrm{~cm}$.

The heat pipe was connected to a pressure transducer (Gems 3500, 0-160 $\mathrm{kPa}$ ) and to an aluminium needle valve, used in turn to vacuum the PHS and 
to fill the PHS with the working fluid. The valve and the pressure sensor were mounted on two orthogonal sides of a rectangular aluminium block previously drilled to create a T-junction. The aluminium block was then glued on the PHP/PHS surface so that the T-junction hole corresponded to the hole on the PHP/PHS auxiliary channel wall, and secured by two bolts passing through the solid part of the PHP/PHS and a counter-plate, as shown in Figure 4. The pressure transducer DC output was sampled at $1 \mathrm{~Hz}$ by a data acquisition system (LabJack U6).

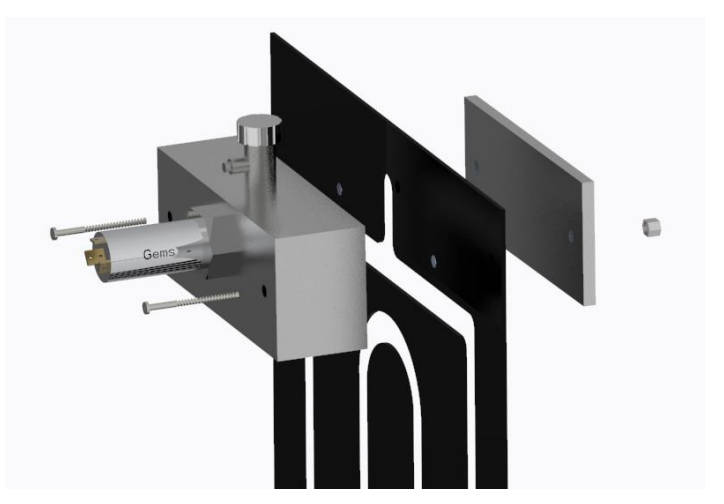

Figure 4: Schematic the pressure transducer and micro-metering valve assembly.

Eight surface thermocouples (Omega Engineering) with response time $\leq 0.3$ $\mathrm{s}$ and flat probe junction were securely fastened between the PHS surface and the clamps, four in the evaporator zone and four in the condenser zone, and connected to a data acquisition system. The temperature distribution in the adiabatic region was monitored by a FLIR infra-red camera (Hti-Xintai A1).

\subsubsection{Procedure}

175 The working fluids used in experiments were de-gassed in a vacuum chamber (Bacoeng) for 24 hours before use. To introduce the working fluid, the PHS modules were vacuumed to a pressure of $0.5 \pm 0.1 \mathrm{kPa}$ (abs) using a twostage vacuum pump (Bacoeng); then, the fluid contained in an external syringe reservoir was slowly driven by the atmospheric pressure into the PHS as the micro-metering valve was gently opened. The filling ratio was $40 \%$ of the total PHP/PHS volume $(1.4 \mathrm{~mL}, 1.9 \mathrm{~mL}$ and $2.6 \mathrm{~mL}$, respectively, for the 3-turns, 5 -turns, and 7-turns PHP/PHS); the filling ratio was determined based on preliminary tests to ensure a maximum pressure of approximately 1.5 bar (abs).

Experiments were conducted by applying to the evaporator section an ascending/descending stepped power ramp ranging approximately between $4 \mathrm{~W}$ and $30 \mathrm{~W}$, and measuring the temperatures on the PHS surface at a sampling rate of $1 \mathrm{~Hz}$. The magnitude of the first step was approximately $6 \mathrm{~W}$ to ensure start-up, based on preliminary tests [7], while the magnitude of subsequent steps was approximately $9 \mathrm{~W}$. For each power step, the heat supply was kept con-

stant until a pseudo steady-state regime was attained, which typically required 
about 30 minutes. Tests were interrupted earlier in case any point of the PHS reached a temperature of $120^{\circ} \mathrm{C}$, to avoid polypropylene softening and/or melting. Preliminary experiments [7] showed the start-up of two-phase circulation in the PHS occurs with a heat input of about $2.5 \mathrm{~W}$ at the evaporator.

The equivalent thermal resistance of the PHS was calculated as:

$$
R=\frac{T_{e v}-T_{\text {cond }}}{\dot{Q}}
$$

where $T_{e v}$ and $T_{\text {cond }}$ are the averages of the four temperature measurements of the PHS surface at the evaporator and the condenser, respectively, in the pseudo steady-states corresponding to each level of the power input, $\dot{Q}$. For the sake of comparison with the thermal resistance of the bare composite polypropylene sheet, one test was carried out using a vaccumed PHS module, without working fluid.

For each of the channel geometries considered (see Figure 21, experiments were carried out for three PHS orientations: (i) vertical, (ii) inclined at $45^{\circ}$, (iii) horizontal. Each test was carried out using a fresh PHS unit to ensure homogeneous experimental conditions.

\section{Results and discussion}

\subsection{Response to a stepped power ramp}

An initial qualitative visualization of the PHP/PHS response to an ascending/descending stepped power ramp supplied to the evaporator is given by FLIR images of the adiabatic region, which are displayed in Figure 5 for PHP/PHS in straight vertical arrangement and with 3 -turns, 5 -turns, and 7 -turns serpentine channel, respectively.

Upon increasing the thermal power supply, one can observe the number of active (i.e., heated) sections of the serpentine channel grows, as well as the active 215 length of each section. As the number of turns increases, there is a more uniform distribution of the thermal power on the PHP/PHS surface. When the thermal power supply is progressively decreased, both the number of active sections and their active length decrease, however the thermal inertia of the system is not negligible and induces hysteresis. In the case of the 3-turn channel, the full activation of all sections occurs during the first step of the descending ramp, long after the maximum thermal power supply has been reached.

Figure 6 shows temperatures measured in the evaporator and in the condenser zones of the 3-turns PHS during the ascending/descending heat supply ramp, for the three heat transfer fluids considered and different orientations, 225 while the corresponding absolute pressures in the serpentine channel are displayed in Figure 7. Similar results were obtained for the 5-turns and the 7-turns PHS. In several cases, the maximum heating power could not be maintained until the system reached a pseudo-steady state because the temperature gradient was too steep (Figure 6), therefore the evaporator temperature could locally approach or exceed the limit imposed by the material. 


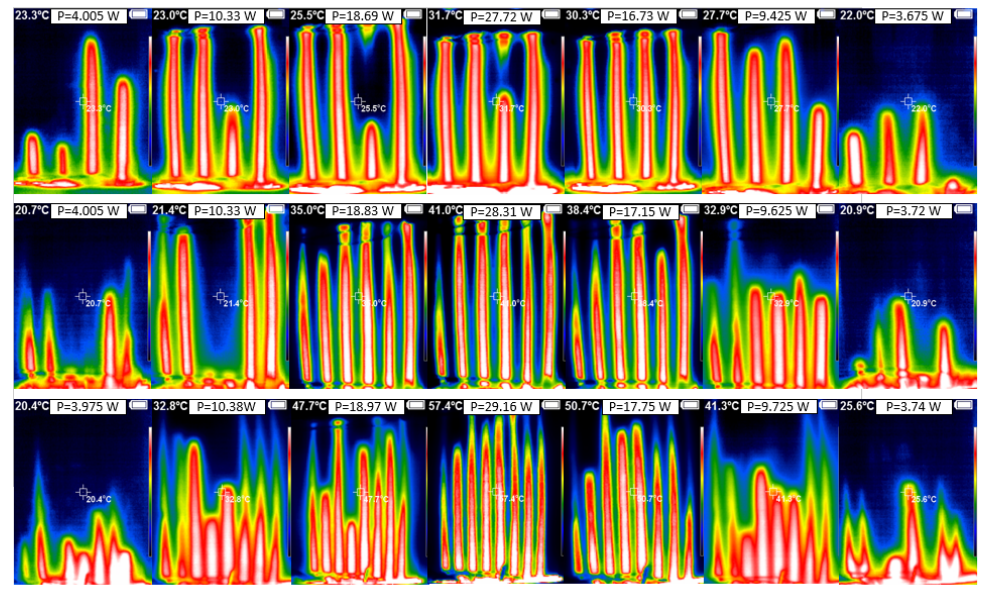

Figure 5: FLIR snapshots of the adiabatic region of a 3-turns (top), 5-turns (center), and 7-turns (bottom) PHP/PHS with vertical orientation and containing FC-72, during an ascending/descending stepped heating power ramp supplied to the evaporator.

At low heating power supply, the temperature measured in different points of the evaporator zone surface is uniform, while as the heating power supply is increased one can observe a significant difference between measurements taken in correspondence of different points of the serpentine channel, with the lower temperatures measured in correspondence of the sections of the channel with stronger fluid circulation. Similar considerations apply to local temperatures measured on the surface of the condenser zone.

The pressure measured inside the serpentine channel (Figure 7) qualitatively mirrors the trend observed for temperature measurements, although it displays a somewhat smoother response to step changes in the heating power supply. At the end of each test (2.5 to 5 hours), one can observe a small increase of pressure with respect to the beginning, which could be due to (i) gas permeation through the polymer channel walls, (ii) potential imperfections in manufacturing (iii) micro-leaks in the connection with the valve and the pressure sensor, 245 (iv) the viscoelastic relaxation of the polymer channel walls $[8$. Whilst this minor pressure increase does not affect significantly the device performance within the duration of a single test, it represents an obvious issue over long periods of time. However, the use of gas barrier layers such as metallic coating was deliberately avoided since the purpose of the present experiments was to evaluate the thermal performance increase of the PHP/PHS with respect to the raw polymer material. At present, there is only one study investigating the performance loss of a polymeric pulsating heat pipe due to gas permeation [13], however a direct comparison with the present results is not possible because the materials used in the two studies (low-density polyethylene and polypropylene, respectively), 255 have different permeation coefficients. 
(i)
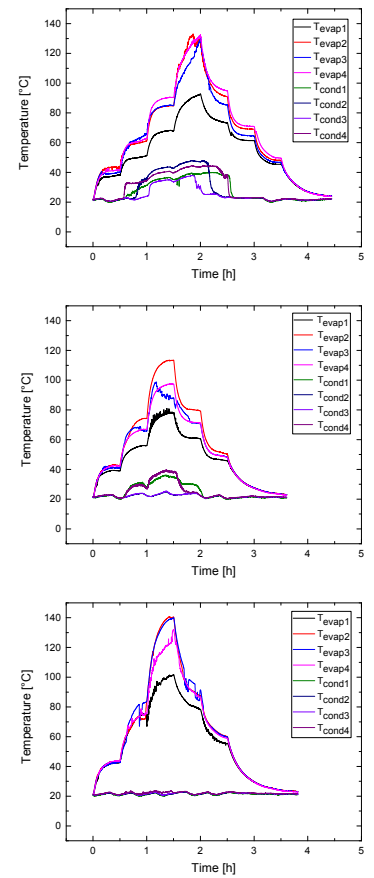

(ii)
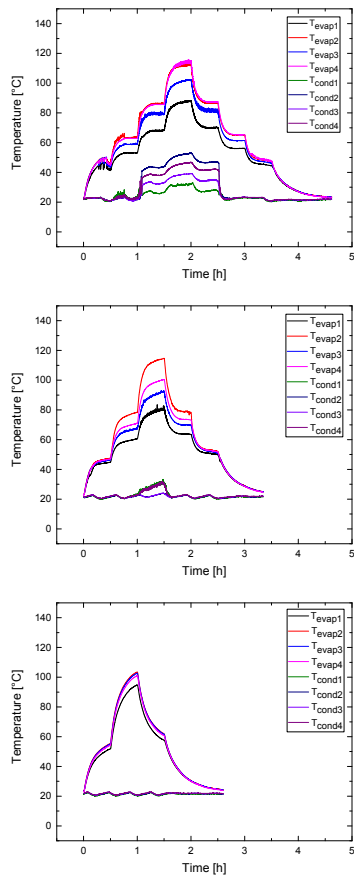

(iii)

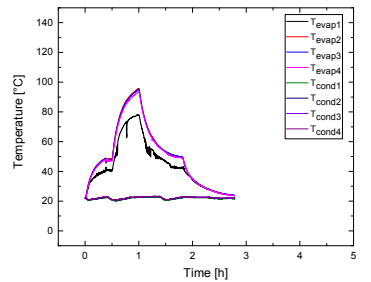

(a)

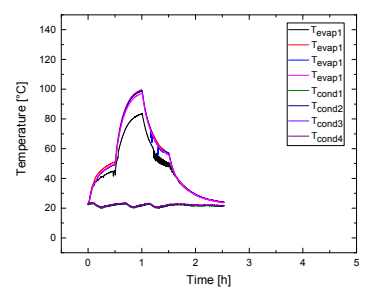

(b)

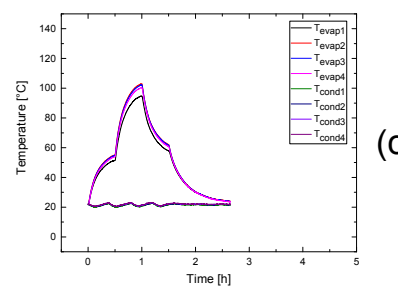

Figure 6: Temperatures measured at the evaporator and the condenser of a 3-turns PHP containing FC-72 (a), ethanol (b), water (c), with vertical (i), $45^{\circ}$ (ii), and horizontal (iii) orientation, during an ascending/descending stepped heating power ramp supplied to the evaporator. The power levels are $4 \pm 0.5 \mathrm{~W}, 10 \pm 0.5 \mathrm{~W}, 18 \pm 0.7 \mathrm{~W}$ and $28 \pm 0.8 \mathrm{~W}$.

\subsection{Effect of design parameters}

The performance of the PHP/PHS achieved with different combinations of the design parameters was estimated based on the values of the equivalent thermal resistance, defined by Eq. (3). Figure 8 displays the measured equivalent thermal resistance as a function of the heating power supply at the evaporator during an ascending/descending heating power ramp, for all the combinations of design parameters considered. With the exception of the horizontal PHP/PHS, the equivalent thermal resistance decreases exponentially with increasing heating power.

In the case of FC-72 and ethanol, and inclinations of $45^{\circ}$ and vertical, the measured values of the equivalent thermal resistance are identical within the experimental error, both during the ascending and during the descending heating power supply ramps. In these cases, the PHP/PHS attain the overall minimum equivalent thermal resistance for a heating power supply of about $30 \mathrm{~W}$, which has a magnitude four to five times smaller than the equivalent thermal resistance measured in the absence of heat transfer fluid $\left(10.8^{\circ} \mathrm{C} / \mathrm{W}\right)$. This corresponds to a four- to five-fold increase of the PHP/PHS thermal performance compared to 
(i)
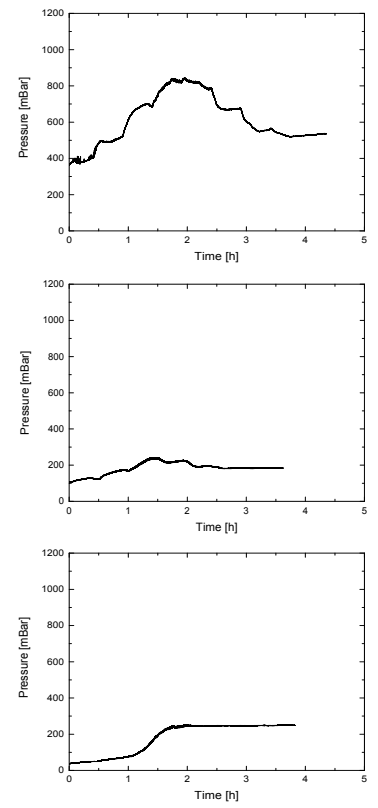

(ii)
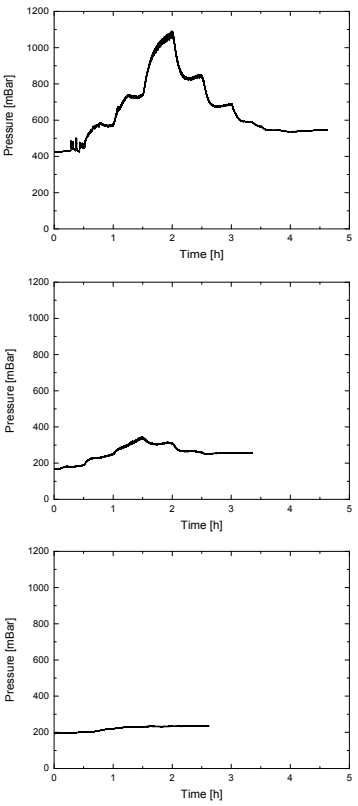

(iii)

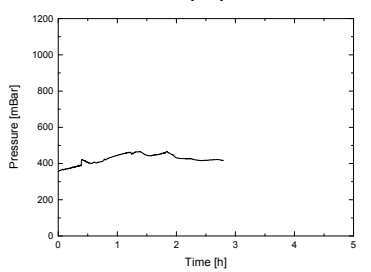

(a)

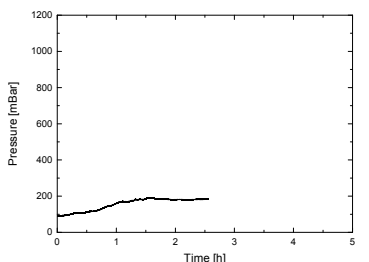

(b)

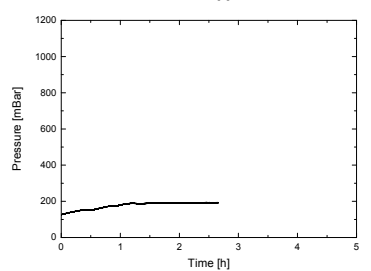

Figure 7: Pressure measured in a 3-turns PHP containing FC-72 (a), ethanol (b), water (c), with vertical (i), $45^{\circ}$ (ii), and horizontal (iii) orientation, during an ascending/descending stepped heating power ramp supplied to the evaporator.

a composite polypropylene sheet having the same composition, size, thickness and weight, in agreement with previous results [8, 11, 12.

In the case of water, the magnitude of the equivalent thermal resistance is much closer to the value measured without heat transfer fluid, and does not exhibit a clear monotonically decreasing trend as the heating power supply is increased. This can be explained with the low saturation pressure, which requires a high vacuum in the serpentine channel, and to the large latent heat, which requires large heating power supplies hence larger temperatures at the evaporator in order to produce the amount of vapour necessary to activate the liquid-vapour mixture circulation. Since in polymeric PHP/PHS the maximum temperature at the evaporator is limited by the melting point of the polymer material, these results suggest water is not a suitable heat transfer fluid for most polymeric heat pipes (except those built with high temperature resistant polymers, of course).

Irrespective of the fluid used and of the number of turns of the serpentine channel, the equivalent thermal resistance measured for the horizontal PHP/PHS increases or remains approximately constant when the heating power 290 supply is increased. The evaporator temperature approaches or attains the upper limit imposed by the material already during the second step of the heating 
(i)
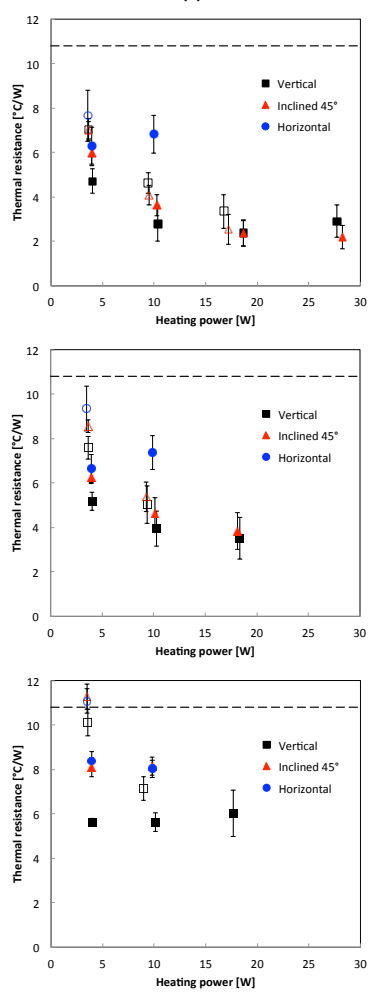

(ii)
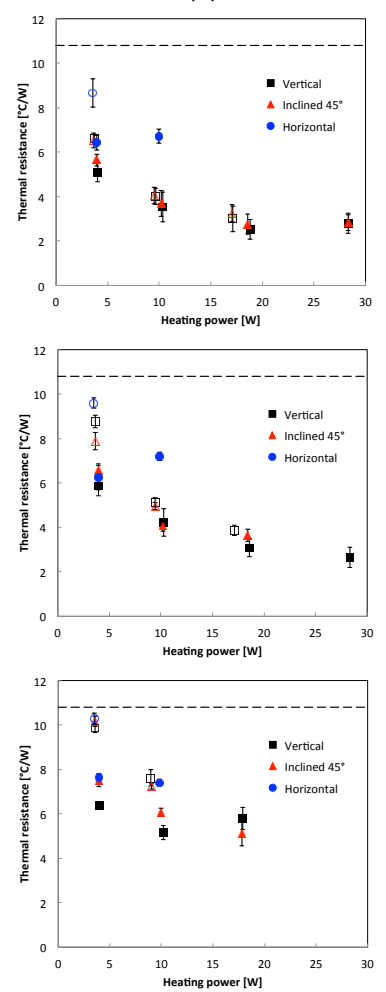

(iii)

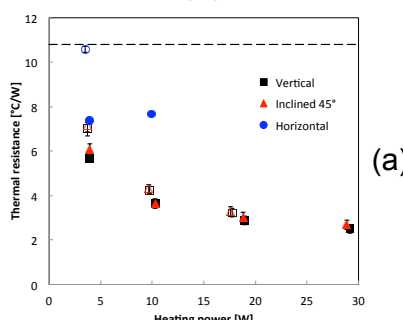

(a)
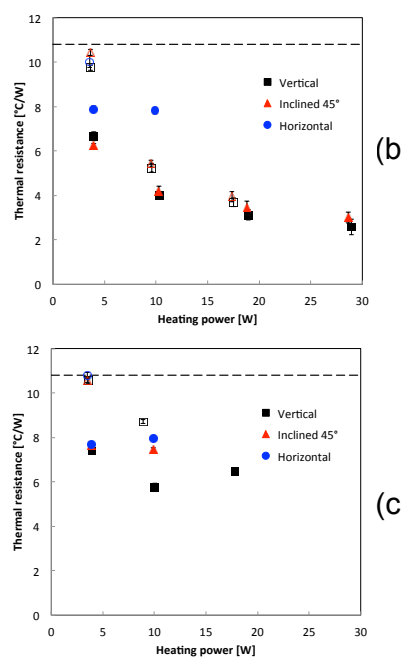

Figure 8: Equivalent thermal resistance of PHP/PHS containing FC-72 (a), ethanol (b), water (c), with a 3-turns (i), 5-turns (ii), and 7-turns (iii) serpentine channel, during an ascending (filled symbols)/descending (open symbols) stepped heating power ramp supplied to the evaporator. The discontinuous horizontal line corresponds to the equivalent thermal resistance of the device without heat transfer fluid. Error bars represent the root mean squared standard deviations of the four temperature measurements, respectively at the evaporator and at the condenser.

power $\operatorname{ramp}(\approx 10 \mathrm{~W})$. During the descending step, the equivalent thermal resistance often attains the value measured in the absence of heat transfer fluid because of the relatively large thermal inertia of the system. This suggests the capillary forces are not sufficiently large to drive the condensate back to the evaporator, reducing significantly the circulation of the liquid-vapour mixture hence the heat transfer performance.

These results are in contrast with the standard design guidelines discussed above, which prescribe the upper and lower limits for the Bond number to ensure the capillary-driven circulation of the liquid in the serpentine channel $(0.4<B o<4)$. In particular, if the Bond number is calculated as $B o=$ $g\left(\rho_{l}-\rho_{v}\right) D_{H}^{2} / \sigma$, the capillary-driven circulation of FC-72 and ethanol should be possible in a channel having a width of $5 \mathrm{~mm}$ (Figure 1a), while if the Bond 
number is calculated as $B o=g\left(\rho_{l}-\rho_{v}\right) D_{H}^{2} / \sigma \cos \theta$ to account for wettability, the suitable fluids are water and ethanol (Figure $1 \mathrm{~b}$ ). However, the results displayed in Figure 8 suggest none of the fluids considered are suitable to ensure the correct operation of the device when the condensate circulation is not assisted by gravity.

Geometric design parameters that can hamper the condensate circulation 310 are the length of the channel sections connecting the evaporator and the condenser $(\approx 200 \mathrm{~mm}$ in this case), which is proportional to frictional losses, and the relativey large aspect ratio of the channel cross-section, which in this case was 1:7. An additional factor that may affect the capillary-driven circulation of the liquid is the possible entrapment of the condensate in the dead volume between the serpentine channel and the fittings with the micro-metering valve and the pressure transducer. In any case, one should bear in mind that the limits on the Bond number were established on the basis of boiling and condensation experiments in channels made of high surface energy materials (metals) at relatively high heat fluxes, while the number of studies about boiling and condensation in channels made of low surface energy materials (such as polymers) at low heat fluxes is not significant. This requires further investigation, which may potentially lead to identify an alternative range of Bond numbers applicable to polymeric channels.
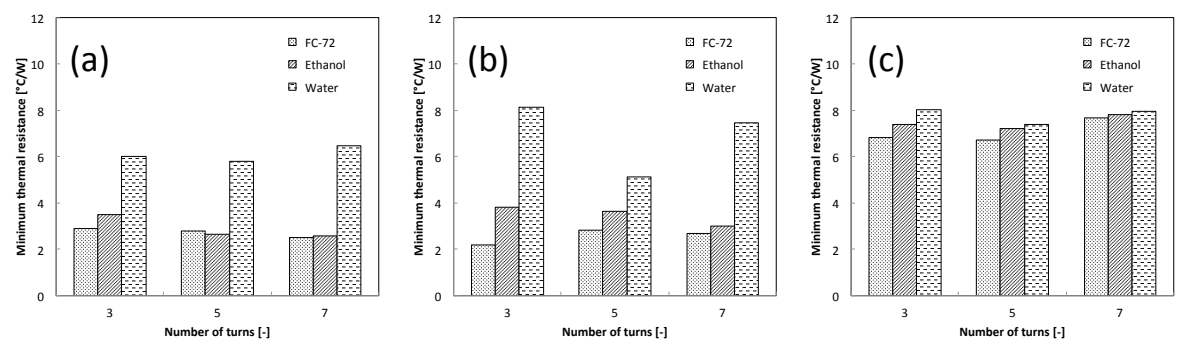

Figure 9: Effect of the heat transfer fluid in a PHP/PHS with vertical (a), inclined at $45^{\circ}$ (b), and horizontal (c) orientation.
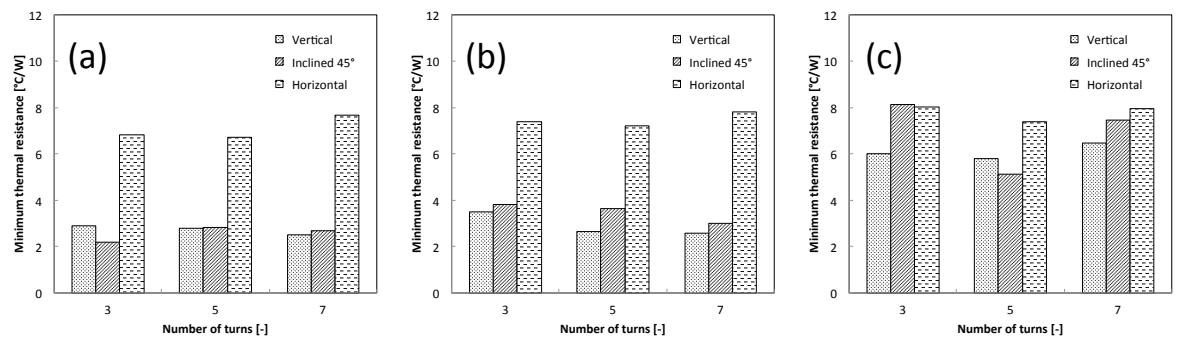

Figure 10: Effect of orientation on a PHP/PHS filled with FC-72 (a), ethanol (b), and water (c). 

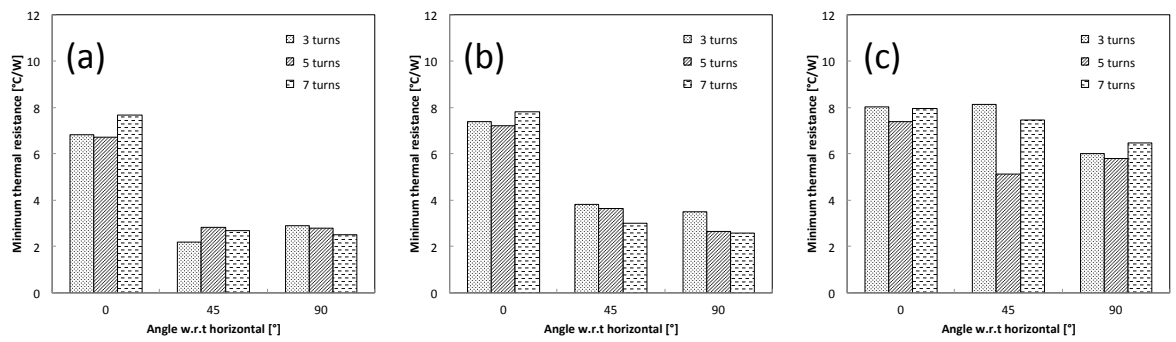

Figure 11: Effect of the number of turns of the serpentine channel on a PHP/PHS filled with FC-72 (a), ethanol (b), and water (c).

Figures 911 display the minimum equivalent thermal resistance of PHP/PHS, defined as the equivalent thermal resistance measured at the maximum thermal power supply for a given set of design parameters. This quantity is representative of the overall performance of the device, therefore it can be used to compare devices having different design parameters.

The effect of the heat transfer fluid used (FC-72, ethanol or water) is displayed in Figure 9, for different orientations of the PHP/PHS with respect to gravity. In most cases, one can observe a systematic effect of the working fluid, and the lowest equivalent thermal resistance is achieved in devices containing FC-72, with ethanol closely following. The absolute minimum thermal resistance measured in this set of experiments is just above $2{ }^{\circ} \mathrm{C} / \mathrm{W}$, which represents an increase of the equivalent thermal conductivity with respect to a polypropylene sheet with the same composition and size of $500 \%$.

Figure 10 shows the effect of the PHP/PHS orientation with respect to gravity, for different heat transfer fluids. For FC-72 and ethanol (Figures 10a and $10 \mathrm{p}$ ), there is a clear performance gap between the vertical or inclined orientations and the horizontal orientation, which confirms gravity is necessary to assist the fluid circulation as discussed above. Finally, the effect of loop geometry (i.e., the number of turns of the serpentine channel) on the PHP/PHS performance is shown in Figure 11. In all cases, there seems to be no significant effect of the number of turns, with the exception of the PHP/PHS containing 345 ethanol with vertical and inclined orientations (Figure 11p), where one can observe a modest but systematic reduction of the minimum equivalent thermal resistance as the number of turns increases.

\section{Conclusions}

The thermal performance of a polypropylene flat pulsating heat pipe (or 350 "pulsating heat stripes") was investigated experimentally to better understand the effects of three design parameters: the heat transfer fluid, the orientation with respect to gravity, and the number of turns of the serpentine channel, respectively. 
The relatively low maximum service temperature in the evaporator zone limits the maximum heat transfer rate (about $30 \mathrm{~W}$ in this case). Thus, fluids with a large latent heat such as water are not suitable heat transfer fluids in this application. Significantly better performances can be obtained using heat transfer fluids having a low latent heat, such as ethanol or perfluorohexane FC-

36072 . In this case, the equivalent thermal conductance of the device can increase up to five times in comparison with that of a polypropylene sheet of the same dimensions.

Irrespective of the heat transfer fluid used, gravity plays a major role in driving the fluid circulation in the serpentine channel, despite the hydraulic diameter was selected so that the Bond number falls within the range commonly prescribed to ensure capillary-driven fluid circulation. This aspects remains ill understood and requires further investigation.

\section{Acknowledgments}

O. Der acknowledges a YLSY doctoral studentship from the Republic of 370 Turkey, Ministry of National Education. A. A. Alqahtani acknowledges a doctoral studentship from the Saudi Ministry for Education. Financial support from the European Space Agency (ESA MAP Project TOPDESS) is gratefullly acknowledged.

\section{References}

375 [1] P. Ruch, T. Brunschwiler, W. Escher, S. Paredes, B. Michel, Toward fivedimensional scaling: How density improves efficiency in future computers,

1. IBM Journal of Research and Development 55 (5) (2011) 15:1-15:13. doi: 10.1147/JRD . 2011.2165677

[2] L. L. Vasiliev, Heat pipes in modern heat exchangers, Applied Thermal Engineering 25 (2005) 1-19.

[3] H. Ma, Oscillating Heat Pipes, Springer, New York, 2015.

[4] S. Das, V. Nikolayev, F. Lefevre, B. Pottier, S. Khandekar, J. Bonjour, Thermally induced two-phase oscillating flow inside a capillary tube, International Journal of Heat and Mass Transfer 53 (19) (2010) 3905 - 3913.

385 doi:https://doi.org/10.1016/j.ijheatmasstransfer.2010.05.009 URL http://www.sciencedirect.com/science/article/pii/ S0017931010002437

[5] M. J. Nine, M. R. Tanshen, B. Munkhbayar, H. Chung, H. Jeong, Analysis of pressure fluctuations to evaluate thermal performance of oscillating heat pipe, Energy $70 \quad(2014) \quad 135$ - 142. doi:https://doi.org/10.1016/j.energy.2014.03.098.

1) URL http://www.sciencedirect.com/science/article/pii/ S0360544214003624 
[6] H. Yang, S. Khandekar, M. Groll, Operational limit of closed loop pulsating heat pipes, Applied Thermal Engineering 28 (1) (2008) 49 - 59. doi:https://doi.org/10.1016/j.applthermaleng.2007.01.033. URL http://www.sciencedirect.com/science/article/pii/ S1359431107000683

[7] O. Der, V. Bertola, M. Marengo, A low cost, flexible pulsating heat pipe technology, in: 3rd Thermal and Fluids Engineering Conference (TFEC), no. TFEC-2018-21455, 2018.

[8] O. Der, M. Marengo, V. Bertola, Thermal Performance of Pulsating Heat Stripes Built With Plastic Materials, Journal of Heat Transfer 141 (9), 091808. arXiv:https://asmedigitalcollection.asme.org/ heattransfer/article-pdf/141/9/091808/6418956/ht\_141】_09\ _091808.pdf doi:10.1115/1.4041952. URL https://doi .org/10.1115/1.4041952

[9] H. Potente, O. Karger, G. Fiegler, Laser and microwave welding - the applicability of new process principles, Macromolecular Materials and Engineering 287(11) (2002) 734-744. doi:10.1002/mame.200290002

[10] A. Humbe, P. Deshmukh, C. Jadhav, S. Wadgane, Review of laser plastic welding process, International Journal of Research in Engineering Technology.

[11] O. Der, M. Marengo, V. Bertola, Pulsating heat stripes: A composite polymer sheet with enhanced thermal conductivity, in: Proc. 16th UK Heat Transfer Conference (UKHTC2019), no. UKHTC2019-082, 2019.

[12] O. Der, S. Edwardson, M. Marengo, V. Bertola, Engineered composite polymer sheets with enhanced thermal conductivity, IOP Conference Proceedings: Materials Science and Engineering 613 (2019) 012008.

[13] J. Lim, S. J. Kim, Fabrication and experimental evaluation of a polymer-based flexible pulsating heat pipe, Energy Con-

1. version and Management 156 (2018) 358 - 364. doi:https: //doi.org/10.1016/j.enconman.2017.11.022.

425 URL http://www.sciencedirect.com/science/article/pii/ S019689041731066X

[14] Y. Zhang, A. Faghri, Advances and unsolved issues in pulsating heat pipes, Heat Transfer Engineering 29 (1) (2008) 20-44. arXiv:https://doi.org/ 10.1080/01457630701677114, doi:10.1080/01457630701677114 URL https://doi .org/10.1080/01457630701677114

[15] M. Mameli, M. Marengo, S. Khandekar, Local heat transfer measurement and thermo-fluid characterization of a pulsating heat pipe, International Journal of Thermal Sciences 75 (2014) 140-152. 
[16] G. B. Wallis, One-dimensional Two-phase Flow, McGraw Hill, 1969.

435

$$
\text { II }
$$

doi:10.1115/1.3447155.

URL https://doi .org/10.1115/1.3447155

440

[18] I. Chernobylskii, I. Tananaiko, Heat exchange during boiling of liquids in narrow annular tubes, Soviet Phys. Tech. Phys. 1 (10) (1956) 2244-2249.

[19] E. Ishibashi, K. Nishikawa, Saturated boiling heat transfer in narrow spaces, International Journal of Heat and Mass Transfer 12 (8) (1969) 863-893. doi:https://doi.org/10.1016/0017-9310(69)90153-7. UR 0017931069901537

[20] D. Bondurant, J. Westwater, Performance of transverse fins for boiling heat transfer, Chem. Engng Prog. Symp. Ser. 67 (113) (1971) 30-37.

[21] P. A. Kew, K. Cornwell, Correlations for the prediction of boiling heat transfer in small-diameter channels, Applied Thermal Engineering 17 (8)

1. (1997) 705 - 715. doi:https://doi.org/10.1016/S1359-4311(96) 00071-3

II URL http://www.sciencedirect.com/science/article/pii/ S1359431196000713

[22] M. Marengo, V. S. Nikolayev, Pulsating Heat Pipes: Experimental Analysis, Design and Applications, Vol. 1, World Scientific, 2018, Ch. 1, pp.

1 1-62. arXiv:https://www.worldscientific.com/doi/pdf/10.1142/ 9789813234406_0001, doi:10.1142/9789813234406_0001 URL https://www.worldscientific.com/doi/abs/10.1142/

[23] B. S. Taft, A. D. Williams, B. L. Drolen, Review of pulsating heat pipe working fluid selection, Journal of Thermophysics and Heat Transfer 26 (4) (2012) 651-656. arXiv:https://doi.org/10.2514/1.T3768, doi:10.2514/1.T3768.

465 URL https://doi.org/10.2514/1.T3768

[24] D. Biolè, V. Bertola, A goniometric mask to measure contact angles from digital images of liquid drops, Colloids and Surfaces A: Physicochemical and Engineering Aspects 467 (2015) 149-156.

[25] D. Biolè, M. Wang, V. Bertola, Assessment of direct image processing methods to measure the apparent contact angle of liquid drops, Experimental Thermal and Fluid Science 76 (2016) 296-305. 
[26] J. Israelachvili, Intramolecular and Surface Forces, 3rd Ed., Academic Press, 2011. 